\title{
Multiple Walking Speed-frequency Relations are Predicted by Constrained Optimization
}

\author{
John E. A. Bertram*† and Andy Ruina $\ddagger$ \\ *Department of Nutrition, Food and Exercise Sciences, Florida State University, Tallahassee, \\ FL 32306, U.S.A. and $\ddagger$ Theoretical and Applied Mechanics and Mechanical and Aerospace Engineering, \\ Cornell University, U.S.A.
}

(Received on 17 July 2000, Accepted in revised form on 2 February 2001)

\begin{abstract}
A person constrained to walk at a given speed $v$ on a treadmill, chooses a particular step frequency $f$ and step length $d=v / f$. Testing over a range of speeds generates a speed-frequency $(v-f)$ relationship. This relationship is commonly posited as a basic feature of human gait. It is often further posited that this curve follows from minimum energy cost strategy. We observed that individuals walking under different constraint circumstances - walking to a range of fixed metronome frequencies (fixed $f$ ) or over a range of spaced markers (fixed $d$ )-produce speed-frequency relations distinct from the constrained $v$ relation. We show here that three distinct speed-frequency curves, similar to those observed, are predicted by the assumption that a walking person optimizes an underlying objective function $F(v, f)$ that has a minimum at the preferred gait. Further, the metabolic cost of transport is a reasonable approximate candidate for the function $F$.
\end{abstract}

(C) 2001 Academic Press

\section{Introduction}

Perhaps in part because of ease of measurement, one oft-discussed feature of gait is the "speedfrequency relationship" as determined from individuals walking on a treadmill over a range of speeds (Cavagna \& Franzetti, 1986; Heglund \& Taylor, 1988; Molen et al., 1972a; Ralston, 1958). Treadmill experiments have the benefit that, by imposing a speed of walking, they mimic the natural constraint of an individual trying to get some place in a given amount of time. Thus, it is reasonable to guess that features of gait observed on a treadmill mimic features of gait in a natural environment (i.e. picking a comfortable gait that provides the selected $v$ ). It is commonly

$\dagger$ Author to whom correspondence should be addressed. E-mail: jbertram@garnet.acns.fsu.edu observed that humans use a fairly well-defined set of $v$ and $f$ combinations that one might term the "natural" walking gait (Grillner, 1981; Nilsson et al., 1985; Sutherland et al., 1980; Winter, 1984). A simple synthesis of such measurements over a range of treadmill speeds is the approximate empirical relation (reviewed by Kuo, 2000)

$$
f=C v^{b}
$$

where $C$ is a constant and $b=0.58$ seems to be a good fit for humans over normal walking speeds. Because the speed an individual walks $(v)$ is the product of step length $(d)$ and step frequency $(f)$, so $v=f d$, the speed-frequency relation can be reported as $f=C d^{b / 1-b}$ or $d=C v^{1-b}$.

Why, ab initio, humans move their bodies as they do is not well settled. Coordination patterns might be shaped by some combination of effects 
including evolutionarily imposed neurological constraints, attempts at energy efficiency, speed, reduced pain and joint wear, reduced susceptibility to unpredictable destabilizing forces and reduced motion of visual and vestibular sensors, etc.

Whatever the reason, if one thinks of the $v-f$ curve as somehow fundamental, then it should be reproducible under some range of conditions. That is, we might reasonably expect that the combination of values that satisfied this minimization when one factor is selected (e.g. speed) would hold when a different factor $(f$ or $d$ ) is selected. We set up a simple experiment to check this assumption. We were initially surprised by the result and then were surprised again by the predictive ability of a simple theory.

\section{A Simple Experiment Measuring $v, f$, and $d$}

The range of conditions we considered were three simple constraints. We constrained, one at a time, either speed, step frequency, or step length and measured the individual's freely chosen values for the other two variables. In each condition the subjects were told simply to walk in a manner that felt comfortable under the imposed conditions. Twelve subjects were tested (six male and six female, ranging in age from 19 to 44 years). Information about the subjects is in Table 1 . Constant $v$ walking was a repeat of the standard treadmill experiments. A treadmill (Precor C962I) was set at constant belt speed. Ten belt speeds were used covering a range both above and below the subject's preferred walking speed (from 0.23 to $2.2 \mathrm{~m} \mathrm{~s}^{-1}$ ). Not all subjects were able to walk at $2.2 \mathrm{~m} \mathrm{~s}^{-1}$ and most subjects were measured at only nine speeds. The order of the belt speeds was randomly selected (in terms of faster and slower speeds). Step frequency at each $v$ was measured by timing the duration of two sets of 20 steps after at least $1 \mathrm{~min}$ of walking at that $v$ (for almost all speeds, at extremely high speeds some subjects could not maintain speed for that duration). The timing of the two sets was averaged. Since $v$ was set as treadmill belt speed and $f$ was directly measured, step length could be calculated as $d=v / f$.

Constant $f$ was imposed by asking the subject to walk to a pre-recorded metronome beat played on a personal cassette player. Frequencies were both above and below the subject's preferred step frequency. Ten frequencies were avail-

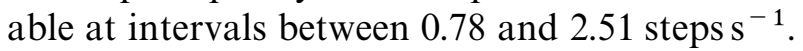

TABLE 1

Physical characteristics of subjects used in this study. Pelvic limb length refers to the linear distance from the ground surface to the lateral tip of the greater trochanter of the femur while the individual stands erect with the footware used in the study. Means for each value (with standard deviation within parentheses) are indicated for the female and male cohorts of the subject group

\begin{tabular}{|c|c|c|c|c|c|c|}
\hline Subject & Sex & $\begin{array}{l}\text { Age } \\
\text { (yr) }\end{array}$ & $\begin{array}{l}\text { Mass } \\
(\mathrm{kg})\end{array}$ & $\begin{array}{l}\text { Height } \\
\text { (m) }\end{array}$ & $\begin{array}{l}\text { Pelvic limb } \\
\quad(\mathrm{m})\end{array}$ & Shoes \\
\hline A & $\mathrm{F}$ & 27 & 56.7 & 1.67 & 0.885 & Yes \\
\hline B & $\mathrm{F}$ & 30 & 63.5 & 1.71 & 0.870 & Yes \\
\hline $\mathrm{C}$ & $\mathrm{F}$ & 40 & 65.8 & 1.57 & 0.850 & Yes \\
\hline $\mathrm{D}$ & $\mathrm{F}$ & 28 & 65.8 & 1.63 & 0.865 & Yes \\
\hline $\mathrm{E}$ & $\mathrm{F}$ & 19 & 66.0 & 1.72 & 0.900 & Yes \\
\hline $\mathrm{F}$ & $\mathrm{F}$ & 23 & 49.9 & 1.63 & 0.877 & Yes \\
\hline Mean (F) & & $27.8(7.1)$ & $61.3(6.6)$ & $1.655(0.056)$ & $0.875(0.017)$ & \\
\hline G & $\mathrm{M}$ & 22 & 67.6 & 1.79 & 0.942 & Yes \\
\hline $\mathrm{H}$ & M & 44 & 89.9 & 1.84 & 0.934 & Yes \\
\hline I & M & 39 & 82.6 & 1.79 & 0.963 & Yes \\
\hline J & $\mathrm{M}$ & 21 & 75.4 & 1.66 & 0.862 & No \\
\hline K & M & 21 & 74.4 & 1.78 & 0.912 & Yes \\
\hline $\mathrm{L}$ & $\mathrm{M}$ & 21 & 68.0 & 1.69 & 0.880 & Yes \\
\hline Mean (M) & & $28.0(10.6)$ & 76.3(8.6) & $1.750(0.069)$ & $0.916(0.039)$ & \\
\hline
\end{tabular}


Each subject walked at nine different frequencies, selected in a subject-specific random order. The subject walked on a level corridor on a $100 \mathrm{~m}$ circuit around a central building core. The subject was then timed over a measured distance twice during the circuit. The two measured $v$ were then averaged. In this case $f$ was set, $v$ measured and step length again calculated as $d=v / f$.

Constant $d$ was imposed by asking subjects to step in line with tape markers arranged at even distances on a level hallway over a $30 \mathrm{~m}$ length. Ten marker spacings were available ranging from 0.225 to $0.935 \mathrm{~m}$ (normal step length for an average individual is approximately $0.56 \mathrm{~m}$ ). Each subject walked on nine of the ten spacings. For each $d$, presented to each subject in a different random order, the duration of 20 steps was timed. This measurement was made for each of two runs per individual and the average of the two was taken. In this case $d$ was set, $f$ measured and speed calculated as $v=f d$.

Step frequency vs. walking speed plots for all subjects in all three constraint conditions are shown in Fig. 1. The data points have been fit using least-squares quadratic regressions (with $f$ as a function of $v$ ) to illustrate the relationship. Visual inspection of these curves indicates three distinct $v-f$ relationships that tend to intersect near the expected preferred walking speed (approximately $1 \mathrm{~m} \mathrm{~s}^{-1}$ ). The difference between the constrained $v$ curves and the constrained $f$ curves are not large, although for all subjects the best-fit constrained $f$ curve consistently has a slightly larger slope. Are the $v-f$ curves significantly different for the three constraint conditions? For each subject in Fig. 1 we report the probability that the data for each pair of conditions could be as depicted without a systematic treatment effect (pair-wise Student's $t$-test). For example, for subject A, the legend " $v$ vs. $d: p=0.001$ " means that there is a 0.001 (or less) chance that the observed difference between the $v$ and $f$ curves in the constant $v$ and constant $d$ experiments could be due to chance alone. The constrained $d$ relations differed significantly from the others in all 24 comparisons. There was a significant difference for 10 of the 12 comparisons between the constrained $v$ and the constrained $f$ cases.

Thus, the $v-f$ curve for walking depends on what is held constant in the experiment. This may be contrasted with the notion of the $v-f$ relation as fundamental to the general circumstances of walking in which case we would expect a common $v-f$ curve for the three constrained situations.

\section{Two Candidate Explanations for the Variety of $\boldsymbol{v}-\boldsymbol{f}$ Curves}

WHAT DETERMINES THE DIFFERENT $v-f$ RELATIONS UNDER THE ALTERNATE CONDITIONS?

The simplest models for the scaling relations observed in walking are those that follow from pendulum-based models. It is well known that these models predict a characteristic $f$, essentially independent of $v$ (Mochon and McMahon, 1980a,b). The scaling laws for non-controlled "passive-dynamic" (e.g. McGeer, 1990) walkers, either determined as dynamic mathematical models or as physical representations, share this constant $f$ feature, as discussed in some detail by Garcia et al. (2000). Although some differences are observed between subjects, the $v-f$ relations are not consistent with the constant frequency predictions of the pendulum-based models.

The second class of possible explanations are based on the three tasks being psychologically distinct. Perhaps one or more of the constraints cause people to move "unnaturally", and therefore the results generated from these situations are not indicative of natural walking. For example, in the fixed $d$ experiments the subjects look down and think, more or less, about each foot placement and this could affect the coordination strategy.

\section{A Third Candidate: Energy Optimization}

Some kind of energy optimization is commonly suggested as a determinant of animal coordination. With this view in mind, various researchers have measured or considered the metabolic cost of transport (oxygen consumption per unit distance) as a function of speed and frequency (or, equivalently, speed and step length or step length and frequency) for human walking. Such studies include Atzler \& Herbst (1927), Elftman (1966), Molen et al. (1972b), Ralston (1958), Zarrugh et al. (1974), Zarrugh \& Radcliffe 

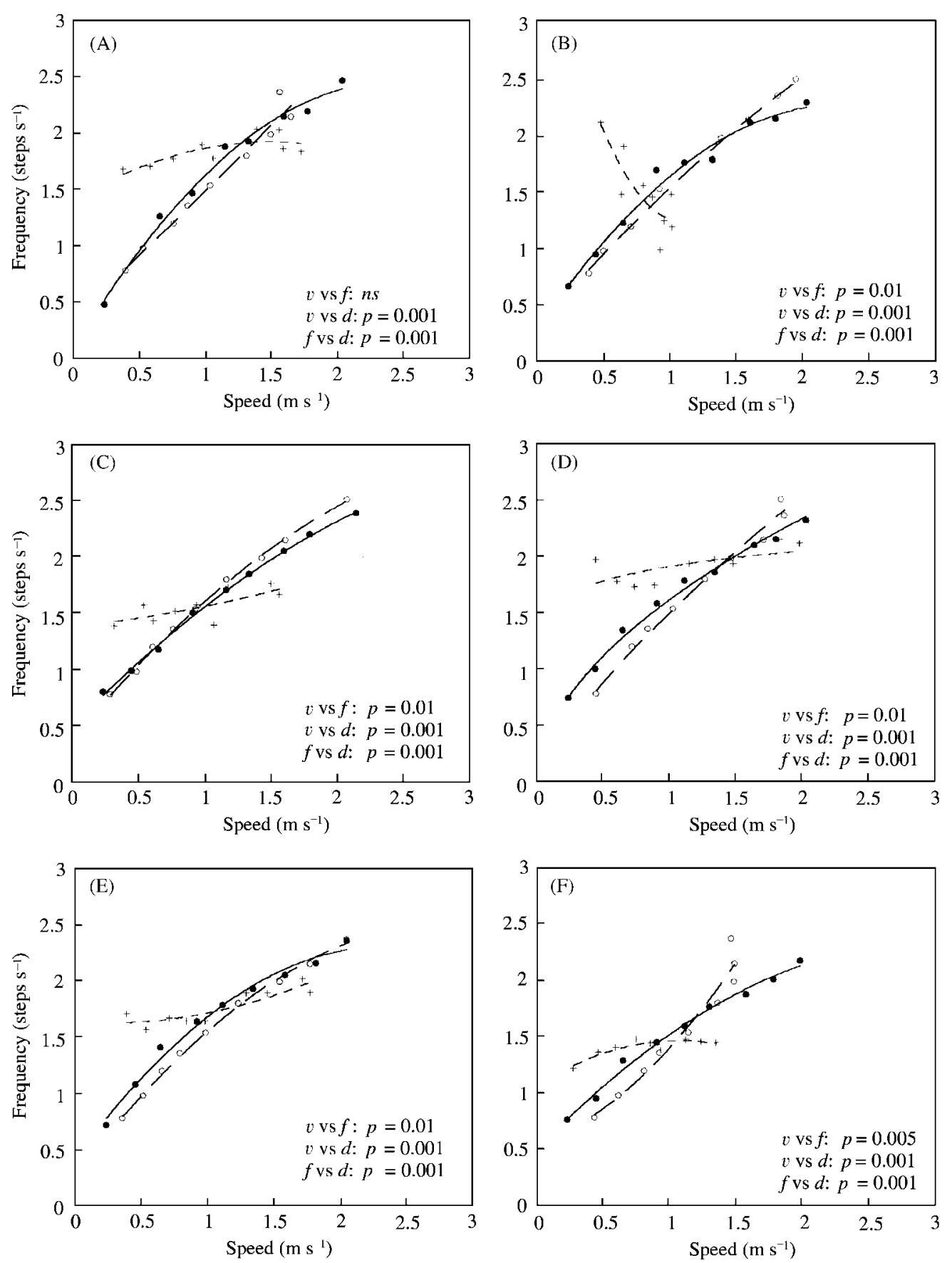

FIG. 1. (A-F): Plots of step frequency $(f)$ against walking speed $(v)$ for all 12 subjects used in this study. The solid circles are speed constrained. The open circles are frequency constrained and the plus signs are step-length constrained. A quadratic is shown for each constraint. For each individual three probability values are given, each showing the result of a pairwise comparison of logarithmic regressions. For instance, for subject A there is no significant difference between the speed constrained $(v)$ and the frequency constrained $(f)$ curves. However, the comparison shows that the step length constrained curve $(d)$ differs from both the others at a probability level of 0.001 . For subject L two frequency constrained studies were conducted, one in which frequency was controlled while the subject was asked to walk a given distance (open circles), and one in which the subject was asked to walk for a given time (open squares).

(1978). This approach is considered in some mechanical detail in Kuo (2000) where good evidence of two types is presented that suggest preferred walking motions at constant $v$ minimize $\mathrm{O}_{2}$ consumption per unit distance. First, Kuo reviews experiments where $\mathrm{O}_{2}$ consumption 

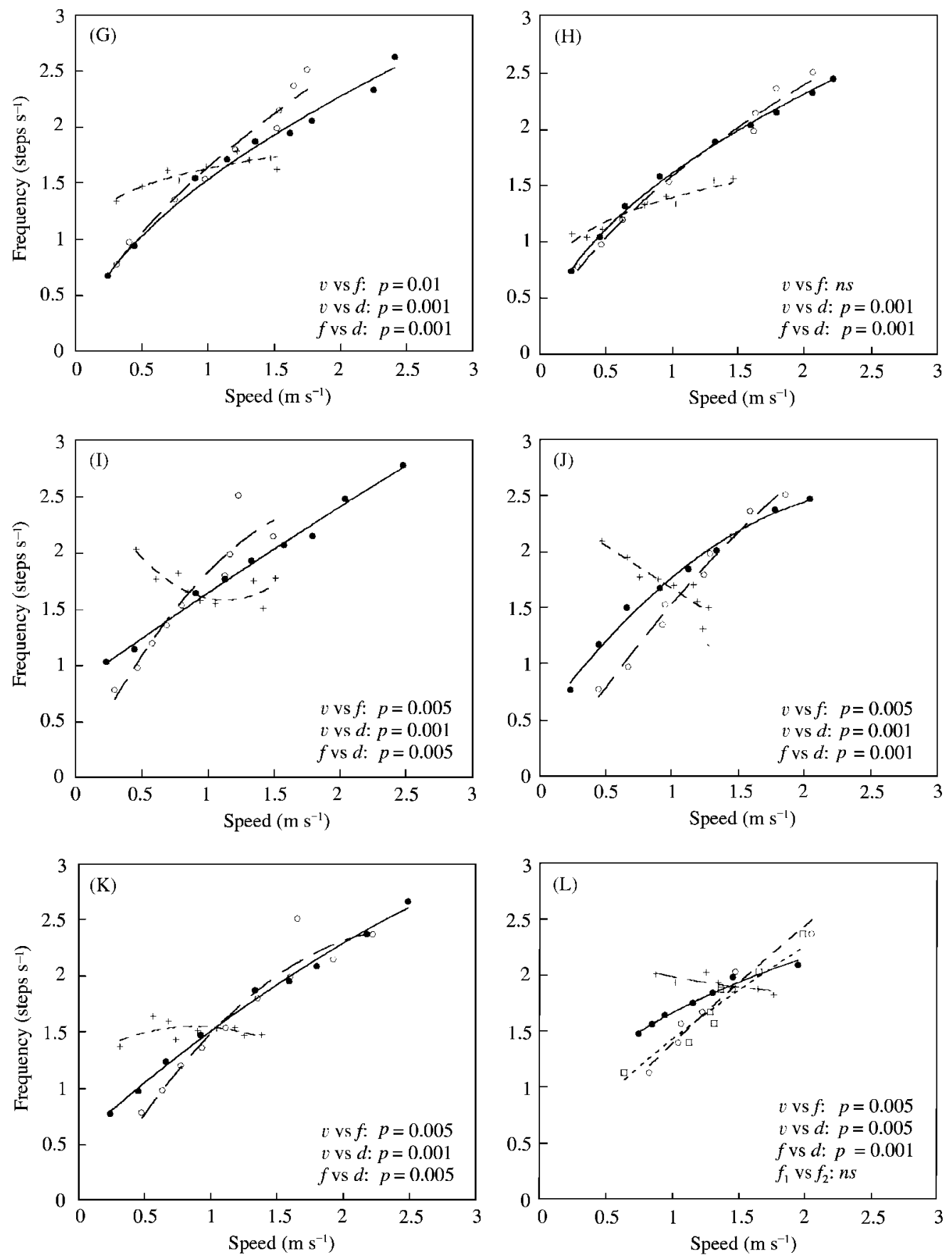

FIG. 1. (G-L).

data are taken from people walking on treadmills at constant speed while adjusting their step frequency to the beat of a metronome. These data can be reduced to a cost $\left(\mathrm{O}_{2}\right.$ consumption rate per unit distance) as a function $(F)$ of $v$ and $f$. Minimizing $F(v, f)$ at a given $v$ very nearly replicates the empirical $v-f$ relation. Second, Kuo (2000) shows that much of the $F(v, f)$ function can be captured by a mechanical model that assigns costs to active toe-off and to oscillating the swing leg faster than its natural pendular frequency.

In general, these energy considerations have been used for the prediction of the preferred speed of walking and the prediction of the preferred frequency at a given speed. 


\section{Constrained Optimization}

The central theoretical concept in this paper is to think of optimizing at a given $v$ as only one of many possible constrained optimizations.

The argument we present is not necessarily energetic, but more generally assumes that there is some scalar (objective) function of the coordination strategy that people tend to minimize to the extent that constraints allow.

Assume an objective function $F$ that is optimized, where $F$ depends on the controllable variables $x_{i}$ needed to adequately describe joint angles and muscle tensions as a function of time during a stride (potentially hundreds or thousands of dynamically changing variables). So $F=F\left(x_{i}\right)$. In a given situation an individual should minimize $F$ subject to the constraints imposed. In the standard treadmill experiment we can think of $v$ as constrained and $f$ as unconstrained and measurable. Most of the $x_{i}$ are unconstrained and at their optimal values so we can think of a reduced objective function $F=F(v, f)$ that depends only on these directly constrained and easily measurable variables.

One could consider as a plausible $F$ the metabolic cost of transport per unit distance. Figure 2 shows contours derived from the metabolic cost surface from one subject in Molen et al. (1972b) (Molen et al. provide detailed data for this individual but report substantial variation between individuals). Each curve indicates the combinations of speed and step frequency (and implicitly step length) that demand equal $\mathrm{O}_{2}$ consumption per unit distance traveled. Each of these contours depicts a cost of walking that is less metabolically costly than the contour that resides outside it. The set of contours emanate from the point of least cost, generally considered to be at about the preferred walking speed and frequency (Cavagna, 1975; Cavagna \& Franzetti, 1986).

Within the optimization paradigm, the standard treadmill experiment is an optimization of $F(v, f)$ with constrained $v$. The optimization prediction for the $v-f$ curve for treadmill walking can be constructed from the objective function $F(f, v)$ and the constraint function $v-C=0$ with the method of Lagrange multipliers (at a constrained optimum the gradient of the cost function is parallel to the gradient of the constraint

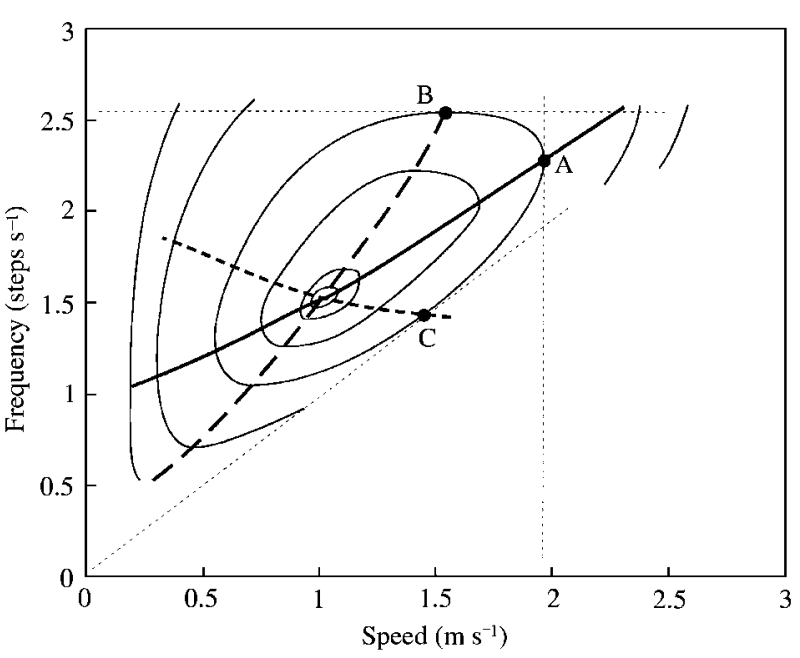

FIG. 2. Contour curves showing constant $\mathrm{O}_{2}$ consumption, above standing still, per unit distance as a function of $v$ and $f\left(\mathrm{ml} \mathrm{O}_{2}\left(\mathrm{~kg}\right.\right.$ body mass $\left.\left.{ }^{-1}\right) \mathrm{m}^{-1}\right)$. The data used to construct these curves are from Molen et al. (1972). The $v-f$ relationships expected from constrained optimization of this function under three conditions are drawn. The curve passing through point A indicates the optimization to constrained speed $(v)$ in which the curve represents vertically oriented tangents to the cost contours. The curve passing through $\mathrm{B}$ indicates optimization to constrained step frequency $(f)$ in which the curve represents horizontally oriented tangents to the cost contours. The curve passing through $\mathrm{C}$ indicates optimization to constrained step length (d) in which the curve represents tangents of the cost contours and lines emanating from the origin (where each line from the origin indicates a different constant step length).

function). For constrained $v$, and the other cases we consider below, the optimization result can also be found by a simple geometric construction (that essentially reproduces the Lagrange multiplier method). For every fixed $v$, the subject is constrained to a line perpendicular to the speed axis in Fig. 2. On a given vertical line, $F$ is then to be minimized. This will be on a level line (metabolic cost contour) that just grazes the constraint line. Otherwise, there would be a smaller $F$ also consistent with the constraint line. Thus, the minimum $F$ for a constrained $v$ is at the point on the $v-f$ plane where a level line of $F$ is tangent with the line $v=$ constant. A sample point A is shown in Fig. 2. The $v-f$ curve is then the sequence of points constructed in like manner, as the points where the level lines of $F$ have vertical tangents.

The $v-f$ curve generated in this manner indicates the optimization solution for the imposed constraint of specified speed. 
Consider now constrained step frequency $f$, as for walking in time to music, a drumbeat or a metronome. What determines the selection of $v$ and $d$ (and the other $x_{i}$ ) in this situation? Taking this as a constrained optimization problem, we look for points like B of Fig. 2. These are points that minimize $F$ on a given line of constant $f$. Using the same reasoning as for constrained $v$ above, point $\mathrm{B}$ is on a level line of $F$ at a point of horizontal tangency. The derived $v-f$ curve for constrained $f$ experiments is generated as a locus of similarly determined points of horizontal tangency.

The last simple situation we consider is that of walking at fixed step length, such as for walking on regularly spaced stepping stones, colored tiles, or railroad ties. The constant $d$ curve on the $v-f$ plane is a line in which $v / f=$ constant. A set of possible $v-f$ points for constant step length is represented by a straight line that passes through the origin in Fig. 2, the slope representing a specific constant step length. Again the minimum $F$ on a constant $d$ line will be at the point of tangency of the constraint line with a level line of $F$, as shown for the typical point $\mathrm{C}$ in Fig. 2. The $v-f$ curve predicted for an optimization to constrained $d$ is generated as a locus of similarly constructed points.

All three of the curves above pass through, and thus intersect at, the minimum of the objective function $F$ at a point one could call the preferred unconstrained speed and frequency.

One could imagine still other constrained experiments that would generate other curves on the $v-f$ plane, but no others seem to correspond to such simple experimental situations.

\section{Summary of the Experiment and Theory}

We have experimentally observed three distinct $v-f$ curves for each subject, depending on constraint, that roughly intersect at a common point near the unconstrained values of $v$ and $f$. The assumption of constrained optimization also generates three curves that, almost independent of the form of $F$ (assuming it is convex and has a minimum for positive $v$ and $f$ ), have similar trends. That is, the frequency constrained relationship has the greatest slope, the speed constrained relationship has a slightly lower slope, and the step length constrained slope differs substantially from the other two, the latter being much closer to zero in the region of preferred walking conditions.

\section{Discussion}

Although we have shown that constrained optimization qualitatively predicts the various $v-f$ curves we cannot demonstrate that this is not an artifact from psychological aspects of the constraint conditions. We can roughly categorize the psychological explanations into three categories.

First, the coordination strategy might not be energetic or cost function based at all. People may have complex control strategies that generate different preferences in different circumstances. The agreement between the optimization model and the preferences that we observe would then be an artifact.

Second, even if energy, say, is optimized it could be that the different constraints alter posture that affect the gait and the energetics. Thus, for constant $d$ walking looking at the ground could alter the energetics and thus contaminate our concept of a single $\mathrm{O}_{2}$ surface applicable to all conditions.

Third, even given the general concept that energy optimization is a governing principle in coordination of locomotion there is no saying what energy a person chooses to minimize. For example, energy per unit time, energy per unit distance, and energy for the task at hand are different objective functions. In nature, one might argue, the task at hand is most often to walk a fixed distance in which case energy for the task and energy per unit distance are equivalent. But if, say, minimization of food use is fundamental people might adapt to tasks where minimization of total energy use is not equivalent to minimization of energy per unit distance. In the metronome constrained test, for example, compare the two tasks of walking for a pre-announced distance or, in contrast, for a pre-announced time. An energy minimizing subject would save total energy by walking with small steps in the fixed time condition. Thus, for the constant $d$ and constant $f$ tests, part of the test could be the subject's perception of whether the task was a fixed distance or a fixed time task. Taking this 
issue into account, it is only the constrained $v$ test that is well defined.

For one subject we conducted a preliminary investigation of the possible effect of task perception. Subject L was given two treatments, both clearly announced to the subject prior to the test: walking a given distance and walking for a given time period. However, we found no significant difference in the $v-f$ curves between these two treatments. Although this one data point is not conclusive, it suggests that any difference does not appear to be overwhelming so that, at least with no significant habituation, the minimization seems not to be at the task level.

Even neglecting these psychological factors the optimization model leaves many open questions. For example, the theory does not indicate whether the selection of a $v-f$ relation in walking is the result of an active optimization, or if it simply follows rules that have been a priori evolved to optimize for a range of contexts general enough to include our experimental conditions. An effect that we did not measure (avoided by limiting the number of repetitions the subjects performed for each circumstance), is how quickly people adapt their condition to the change in constraint, or whether, in fact, the optimization gets better as people practice in the constrained situations.

Although we have shown agreement between the trends predicted by optimization and the choices made by individuals, we have not made a direct comparison between the observed walking $v-f$ behavior of our study subjects and $\mathrm{O}_{2}$ contours for those same individuals. We reserve for further study whether the variations between subjects observable in Fig. 2 correlates with differences between oxygen consumption surfaces.

An individual may seem more commonly to be constrained by the demands of providing a particular walking speed $v$, but the $v-f$ relationship generated from speed constraint is not really any more fundamental than the other $v-f$ relations from the other constraints. The three conditions we tested have counterparts in common experience. There are other constraints that would fall into the realm of natural behavior such as the carrying of weights, walking bent over to clear overhead obstacles, walking in circles, walking with given heel strike (to control sound genera- tion while hunting, say). Would the apparent consistency with optimization still be evident for constraints that fall far outside the personal or evolutionary experience of individuals? Imagine a person told to walk on a treadmill where the belt speed was actively adjusted in response to the subject's motion to follow the relation $f^{2} v=$ constant. Similarly, one could consider walking in reduced gravity, walking with a weight strapped to one leg, etc. Would the subject's most comfortable gait be one predicted by constrained optimization of the same objective function? Would this change with practice?

Molen et al. (1972a,b) observed that the minimum of the metabolic cost function falls at a slightly slower speed than the preferred walking speed.

This could be explained within the optimization theory if the applicable optimization function is not just energetic in nature but also penalizes slowness. For example $F$ might be of a form that rewards speed, such as

$$
F=C_{1}\left(\dot{\mathrm{V}}_{2} / \text { distance }\right)+C_{2}(v)^{-n},
$$

where $C_{1}$ and $C_{2}$ are constants determining the relative weighting of each component. Such a form would reduce the difference between the integrated cost in the fixed distance and fixed time treatments. There is no smooth cost function, however, that has the property that optimization at fixed time and at fixed distance predicts the same $v$ for a given $f$ (both the gradient of $F$ and of $F / v$ would have to be in the direction of the gradient of $f$ at the same $v-f$ pair).

The obvious advantage of being metabolically effective, by some functional measure of effectiveness (i.e. food use per time, or per distance, speed of task performance, etc.), leads to the prediction that people or animals would likely be reasonably effective at the things they do often. We might assume that locomotion, particularly walking, is a behavior that is performed regularly and is of critical importance to the survival of the individual in natural circumstances. The success of the model described here, to the extent that it is not a psychological artifact, supports the claim that this effectiveness includes the behavioral plasticity to remain as effective as possible in a range of circumstances or imposed constraints. 
Gary Sforzo, Jeff Ives and John Sigg at Ithaca College shared their equipment and helped set up the experiments at Ithaca College. Dave Cabrera and Mike Coleman helped to test subjects. Saskya van Nouhuys, David Lee and two anonymous reviewers made comments that usefully improved the manuscript. This work has been funded in part by a NSF Biomechanics Grant (to AR).

\section{REFERENCES}

Atzler, E. \& Herbst, R. (1927). Arbeitsphysiologische studien. Part 3 Pflug. Arch. Ges. Physiol. 215, 291-328.

Cavagna, G. A. (1975). Force platforms as ergometers. J. Appl. Physiol. 39, 174-179.

Cavagna, G. A. \& Franzetti, P. (1986). The determinants of the step frequency in walking humans. J. Physiol. 373, 235-242.

ElFTMAN, H. (1966). Biomechanics of muscle with particular application to studies of gait. J. Bone Jt. Surg. 48A, 363-377.

Garcia, M., Chatterjee, A. \& Ruina, A. (2000). Efficiency, speed, and scaling of two-dimensional passive dynamic walking. Dyn. \& Stab. Syst. 15, 75-99.

GrILlNER, S. (1981). Control of locomotion in bipeds, tetrapods, and fish. In: Handbook of Physiology-The Nervous System (Brooks, V., ed.), Vol. II, pp. 1179-1236.

Heglund, N. C. \& TAYlor, C. R. (1988). Speed, stride frequency and energy cost per stride: How do they change with body size and gait? J. Exp. Biol. 138, 301-318.

KUO, A. (1999). Stabilization of lateral motion in passive dynamic walking. Int. J. Robot. Res. 18, 917-930.

KUO, A. (2000). A simple model of bipedal walking predicts the preferred speed-step length relationship. J. Biomech. Eng. (in press).
Mochon, S. \& MCMAHON, T. A. (1980a). Ballistic walking. J. Biomech. 13, 49-57.

Mochon, S. \& MCMAhon, T. A. (1980b). Ballistic walking: an improved model. Math. Biosci. 52, 241-260.

MCGeER, T. (1990). Passive dynamic walking. Int. J. Robot. Res. 9, 68-82.

Molen, N. H., Rozendal, R. H. \& Boon, W. (1972a). Fundamental characteristics of human gait in relation to sex and locomotion. Proc. Kon. Ned. Akad. Wet. C-75, 215-223.

Molen, N. H., Rozendal, R. H. \& Boon, W. (1972b). Graphic representation of the relationship between oxygen-consumption and the characteristics of normal gait of the human male. Proc. Kon. Ned. Akad. Wet. C-75, 305-314.

Nilsson, J., Thorstensson, A. \& Halbertsma, J. (1985). Changes in leg movements and muscle activity with speed of locomotion and mode of progression in humans. Acta Physiol. Scand. 123, 457-475.

RALSTON, H. J. (1958). Energy-speed relation and optimal speed during level walking. Int. Z. Angew. Physiol. 17, 277-283.

RaWlings, J. O. (1988). Applied Linear Analysis: A Research Tool, 553pp. Pacific Gove: Wadsworth and Brooks.

Sutherland, D. H., Olsen, R., CoOper, L. \& Woo, S. L.-Y. (1980). The development of mature gait. J. Bone Jt. Surg. 62A, 336-353.

WINTER, D. A. (1984). Kinematic and kinetic patterns in human gait: variability and compensating effects. Hum. Movement Sci. 3, 51-76.

Zarrugh, M. Y., Todd, F. N. \& Ralston, H. J. (1974). Optimization of energy expenditure during level walking. Europ. J. Appl. Physiol. 33, 293-306.

Zarrugh, M. Y. \& RADClifFe, C. W. (1978). Predicting metabolic cost of level walking. Europ. J. Appl. Physiol. 38, 215-223. 